\title{
STRUCTURAL PARAMETERS OPTIMIZATION FOR A PROPORTIONAL SOLENOID
}

\author{
Yu, Y. X.; Ke, S. D. \& Jin, K. D. \\ Faculty of Mechanical Engineering \& Automation, Zhejiang Sci-Tech University, Hangzhou, China \\ E-Mail: yyxin@zstu.edu.cn
}

\begin{abstract}
This paper presents an optimization method for the structural parameters of a proportional solenoid. The 3D finite element method simulation model for performance analyse of proportional solenoid was validated by force-displacement characteristic experiment. Parameter sensitivity analysis results showed that the displacement of the armature, the length of the armature, the radius of the armature, the width of the first half of the sleeve and the angle of the magnetic-isolated ring have significant effects on the electromagnetic force. Key structural parameters were optimized according to the simulation results. Experiments results showed the average electromagnetic force was increased by $20.1 \%$ and the effective stroke was extended to $2.1 \mathrm{~mm}$.

(Received in July 2020, accepted in November 2020. This paper was with the authors 1 month for 2 revisions.)
\end{abstract}

Key Words: Optimization, Proportional Solenoid, Force-Displacement Characteristic, Parameter Sensitivity Analysis

\section{INTRODUCTION}

As the key implementation equipment of the electro-hydraulic proportional directional valve, the performance of the proportional solenoid has a direct effect on the entire hydraulic system [1]. When the spool is excessively blocked, the proportional directional valve will be jammed or stuck [2]. Some manufacturers of hydraulic components have enhanced the excitation current to obtain a high electromagnetic force to reduce the probability of jamming. However, coil heating is intensified and reliability is reduced accordingly. The proportional solenoid structure should be optimized to obtain a higher electromagnetic force under fixed excitation to solve this problem effectively.

Hitherto, formula method and finite element method (FEM) have been widely used in solenoid research. Hu et al. [3] developed a simulation system of a solenoid valve by using Visual Basic 7.0 and MATLAB to optimise the design parameters of the solenoid. However, a large number of calculations are required to solve the objective function directly. With the development of computer technology, FEM has gradually improved and has been applied to electromagnetic design. Li [4] and Zhang and $\mathrm{Xu}$ [5] investigated the force-stroke characteristic of a solenoid by 2D FEM. Angadi et al. [6] constructed a model of a solenoid valve by 2D FEM, and the model can predict the heating problem well. Tao et al. [7] considered the effects of the various properties of a soft magnetic material to optimise a solenoid valve by $2 \mathrm{D}$ FEM. Though, they all need to simplify the model to a two-dimensional plane, discard some of the parameters which may have a great impact on the calculation results accuracy. 3D FEM has been widely used to meet the design needs and improve simulation accuracy. Zhou et al. [8] calculated the electromagnetic force by magnetic path division and 3D FEM simulation. The result showed that simulation values are close to measured results. Sun et al. [9] investigated the electromagnetic energy conversion in a solenoid valve by using 3D simulation. They found that coil turns and armature thickness are the two significant parameters. Liu et al. [10] created a 3D model of a high-speed solenoid with a non-axisymmetric structure and analysed the effects between the key parameters based on response surface methodology. When the correlation of these parameters is low, the accuracy of the calculation results will be low relatively. Numerous 
studies have shown that different structural parameters have different effects on the electromagnetic force [11-13]. Furthermore, as the number of parameters increases, the computational amount increases and it is difficult to determine the key parameters [14]. Few scholars have proposed specific optimization methods to obtain the influence of parameters on performance.

In this study, the following optimization targets are considered: (1) the electromagnetic force is increased under the same excitation current and (2) the constant force-displacement characteristic is improved. An optimization method is proposed to achieve these targets. The specific steps of the method are as follows: First, the proportional solenoid structure is analysed, and the 3D FEM model is established and validated. Second, parameter sensitivity analysis is conducted to select the key parameters for analysis, simplify the analysis process. Finally, the parameters are adjusted according to the simulation results. The optimized sample is tested and compared with the simulation results. The experimental results show that the static performance of the proportional solenoid optimized by the proposed method is effectively improved.

\section{METHODS}

In this section, the principle of proportional solenoid, 3D FEM model and experimental set up are presented. The experimental results were used to verify of 3D FEM model.

\subsection{Working principle of proportional solenoid}

Fig. 1 shows a schematic diagram of the proportional solenoid called GP45, which was provided by HOYEA Co., Ltd. [15]. Its total stroke is $2.5 \mathrm{~mm}$, its effective working stroke is $2.1 \mathrm{~mm}$, and its average electromagnetic force is $100 \mathrm{~N}$ at a rated current of $2.5 \mathrm{~A}$. This proportional solenoid structure is made of magnetic and non-magnetic materials. The magnetic components include the sleeve, armature, fixed iron core and shell cover. The non-magnetic materials include the magnetic-isolated sheet, magnetic-isolated ring, plunger, nut and other components. The sleeve is divided into two parts by the magnetic-isolated ring, namely, the first half of the sleeve and the second half of the sleeve. The basin-type pole shoe structure with a cone-shaped end is formed by combining the first half sleeve and the fixed iron core. The excitation control coil is placed between the sleeve and the shell cover. The left end of the armature is connected to the plunger, which is used for outputting the electromagnetic force, whereas the other end of the armature is assembled with a spring and a nut, which is used to reset the armature displacement.

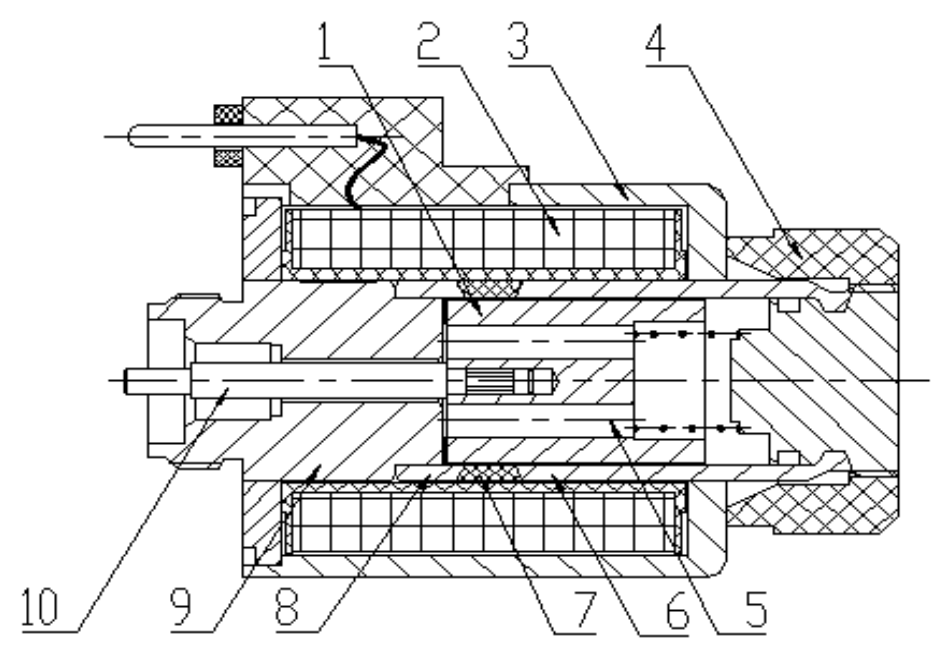

1. armature; 2 . coil; 3 . shell cover; 4. nut; 5 . oil conduit; 6 . second half of the sleeve;

7. magnetic-isolated ring; 8. first half of the sleeve; 9 . fixed iron core; 10. plunger

Figure 1: Schematic diagram of GP45. 
When the coil is excited by a current, two magnetic circuits, namely $\Phi_{1}$ and $\Phi_{2}$, are formed by the special structure of the magnetic-isolated ring [16] as shown in Fig. 2. The electromagnetic force $F_{m 1}$ is generated because the magnetic flux in $\Phi_{1}$ and the magnetic flux in $\Phi_{2}$ drive the armature to generate electromagnetic force $F_{m 2}$. When combined, $F_{m 1}$ and $F_{m 2}$ yield the resultant force $F_{m}$. The variation trends of $F_{m}, F_{m 1}$ and $F_{m 2}$ are shown in Fig. 3. With the movement of the armature, $F m_{1}$ gradually decreases with the increase in armature displacement and $F_{m 2}$ gradually increases with the increase in armature displacement, compensating for the force reduced by $F_{m 1}$. Thus, the total electromagnetic resultant force $F_{m}$ does not vary with the changes in the armature displacement. Theoretically, the forcedisplacement curve is a straight line, which has a great constant characteristic within the working stroke, which is advantageous for proportional control [17, 18].

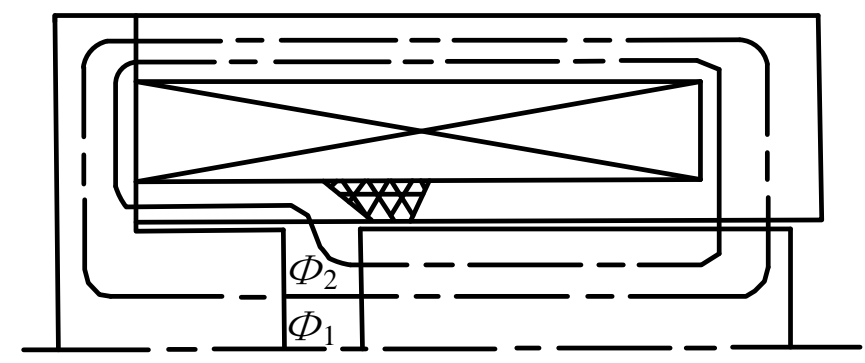

Figure 2: Distribution of magnetic circuits.

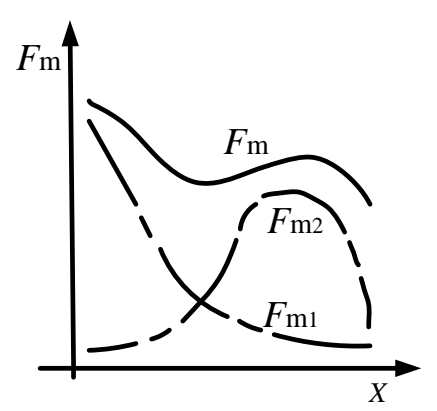

Figure 3: Laws of electromagnetic force.

\subsection{D FEM model and verification}

The simulation 3D FEM Model of proportional solenoid is run on the ANSYS Workbench platform.

\section{Working principle of proportional solenoid}

Structural analysis shows that some components do not have any effect on the magnetic circuit, such as the nut and plunger. The simulation model should be simplified to reduce the number of elements and consequently improve the calculation speed. The sleeve, fixed iron core and shell cover are made of the same magnetic material, which is DT4. In order to improve the simulation accuracy, the magnetisation curve of this is measured and it is shown in Fig. 4 a. These three components have fixed relative distances and are combined into an integral component with a Boolean model. The magnetic-isolated ring, magnetic-isolated sheet, coil frame and nut are made of non-magnetic alloys, and their relative magnetic permeability is the same as that of air; thus, air can be directly used to replace these components.

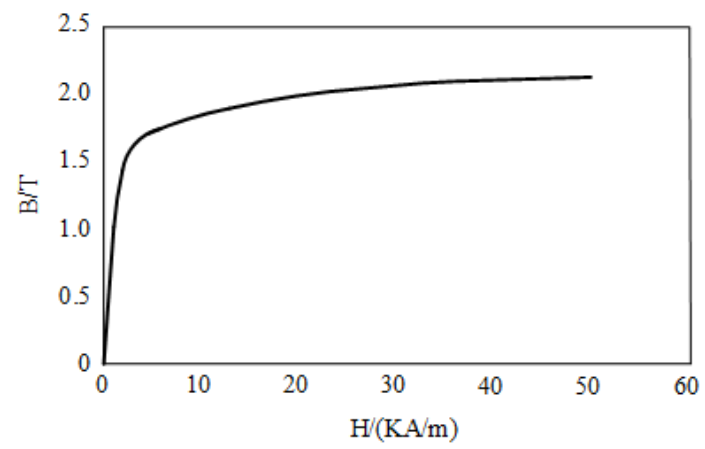

a) Magnetization curve of DT4

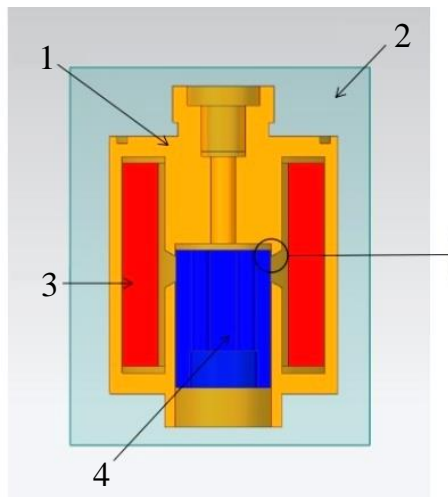

b) Simplified model of GP45

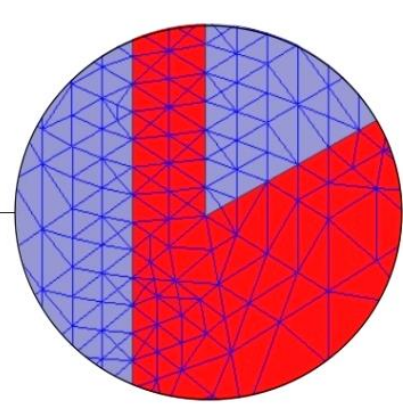

c) Local grid

1 - fixed support; 2 - air boundary; 3 - excitation source; 4 - motion block

Figure 4: Modelling and simulation details. 
In the simplified 3D model (Fig. 4 b), the entire solenoid is divided into three parts, namely, motion block (armature), fixed block (shell cover, iron core, sleeve and other components) and excitation source (direct current [DC] coil). The use of the balloon boundary is a reasonable approach and can significantly reduce the scope of the solution domain; as a result, the memory and central processing unit overheads are minimized. The proportional solenoid is surrounded by rectangular air, and the outer surface of the air is defined as the balloon boundary.

The local grid is magnified in Fig. $4 \mathrm{c}$. Mesh quality directly affects the accuracy of the calculation results; thus, the air gap mesh cells between the sleeve and the armature are densified to more than two layers to reflect the electromagnetic force variation caused by air gap changes accurately. The angle of the magnetic-isolated ring is processed using the same approach. The excitation coil is driven by the DC current, regardless of the skin effect.

\section{Experimental platform}

The mechanical structure of the experimental platform consists of the test bracket, position adjustment lead screw (not shown), proportional solenoid, force transmission device, force sensor, laser displacement sensor, proportional control amplifier and signal generator, as shown in Fig. 5. The host computer is composed of the computer and data acquisition card. The host computer software is developed based on LabVIEW. The static characteristic test of the proportional solenoid can be measured by manually adjusting the lead screw to change the position of force sensor. Fig. 6 shows some experimental samples experimental platform. The specifications of the main sensor equipment of the experimental platform are shown in Table I.

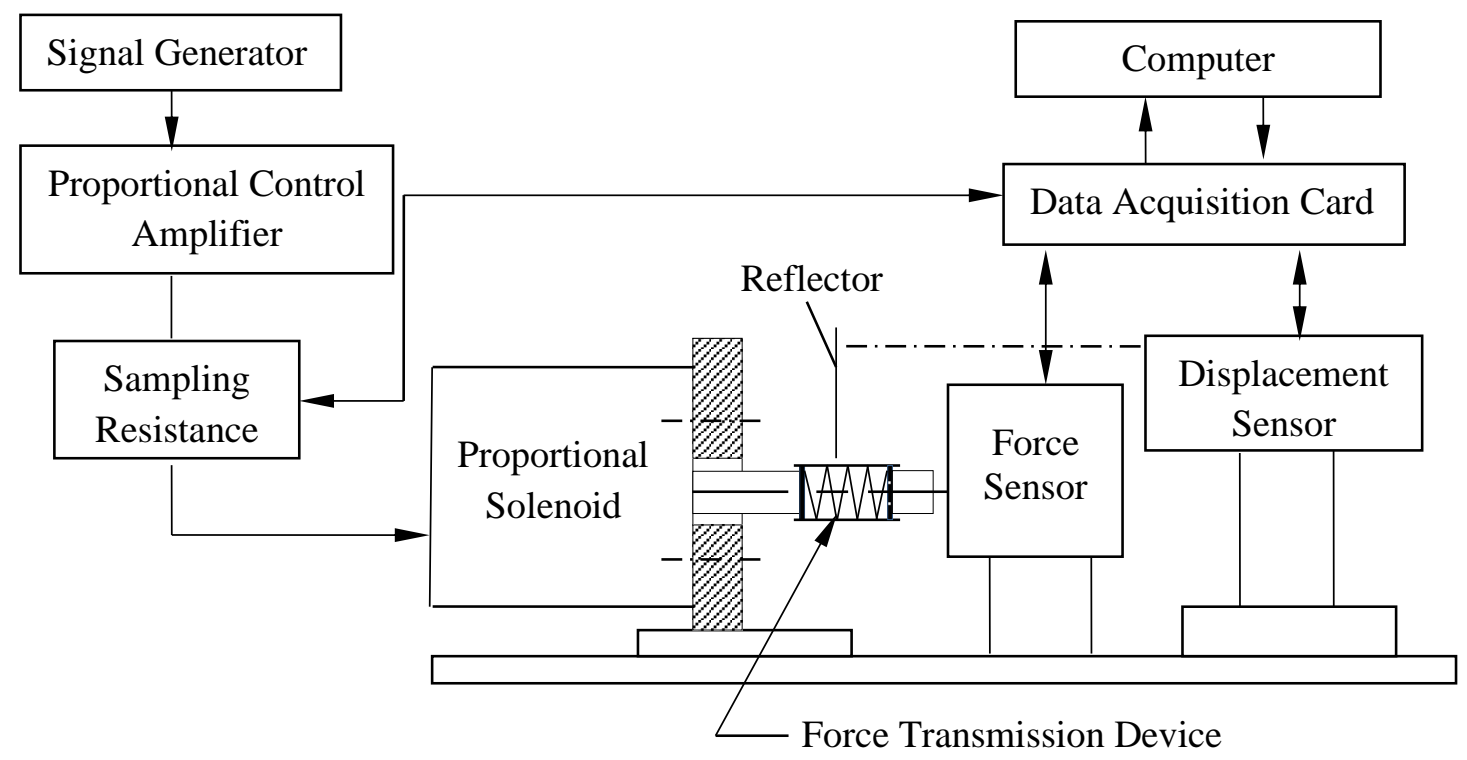

Figure 5: Experimental platform diagram.
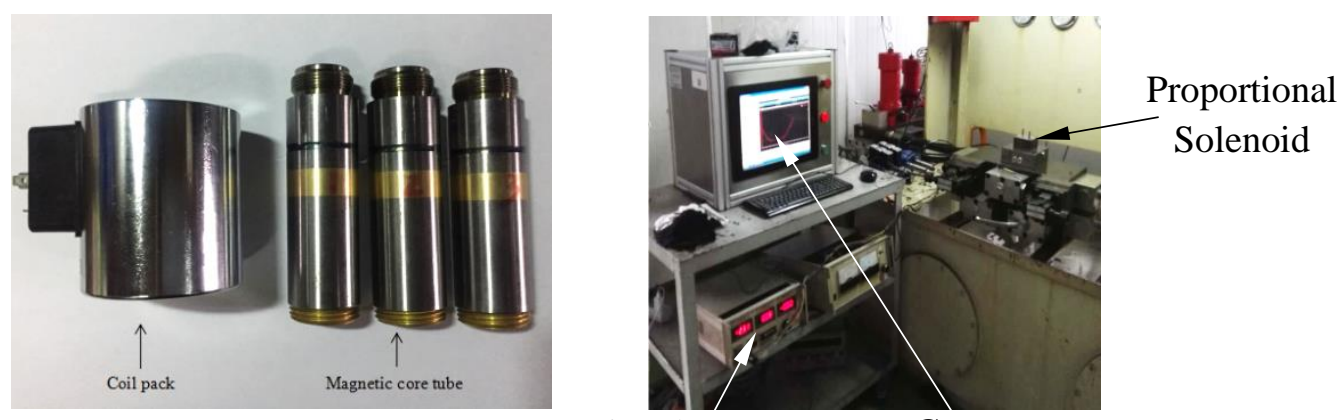

Signal Generator

Computer

Figure 6: Experimental samples and platform. 
Table I: Precision of main equipment.

\begin{tabular}{|l|c|c|}
\hline Main equipment & Force sensor & Displacement sensor \\
\hline Type & C9C-200N & ZLD-S100-10 Laser displacement sensor \\
\hline Producer & HBM & ZSY Group Ltd. \\
\hline Measurement range & $0 \mathrm{~N}$ to $200 \mathrm{~N}$ & $0 \mathrm{~mm}$ to $10 \mathrm{~mm}$ \\
\hline Non-linearity & $<0.2 \%$ & $<0.05 \%$ \\
\hline
\end{tabular}

\section{Verification of 3D FEM model}

For the force-displacement characteristic experiment, the coil current value is fixed and the lead screw is adjusted. The computer records the force of the plunger at different displacement points. This process is performed iteratively to obtain the force-displacement curves at different excitation currents.

The force-displacement curves with different currents obtained from the experiment and from the simulation of original parameters are shown in Fig. 7.

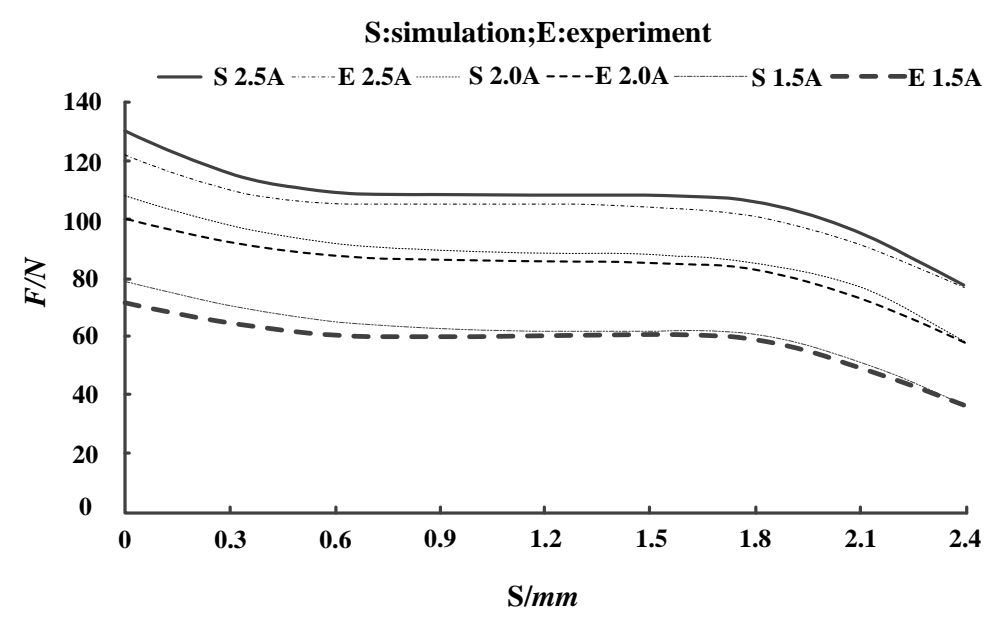

Figure 7: Simulation and experimental results based on the original parameters.

Under different excitation levels, the variation trends of the simulation curves are consistent with those of the experimental curves. However, deviations exist between the experimental results and the simulation results. In addition to the material factor, the deviations may be caused by the simplification of the proportional solenoid model, and this process may have an influence on the calculation. Furthermore, the simulation was conducted under an ideal condition, and certain factors, such as the magnetic leakage, frictions and temperature changes, have been disregarded. Statistical analyses were conducted at each point of errors, and the results are shown in Table II. When the currents are 1.5, 2.0 and 2.5 A, the average errors are $4.4 \%, 4.5 \%$ and $4.0 \%$, respectively. It is clear that this 3D FEM model has enough accuracy to carry out the optimization task.

Table II: Errors between the simulation and experimental results.

\begin{tabular}{|c|c|c|c|}
\hline Current (A) & Average error (\%) & Maximum error (\%) & Minimum error (\%) \\
\hline 1.5 & 4.4 & 9.2 & 0.8 \\
\hline 2.0 & 4.5 & 7.7 & 0.1 \\
\hline 2.5 & 4.0 & 6.9 & 0.5 \\
\hline
\end{tabular}

\subsection{Methods of parameters optimization}

The structural parameters of the proportional solenoid have different effects on the electromagnetic force and considering all structural parameters will cause the model to be 
highly complex and the computation amount will be huge. The parameter sensitivity analysis method can help in the selection of some key parameters and improve the optimization efficiency.

The steps of sensitivity analysis are as follows:

(1) establishing the system model,

(2) selecting the uncertainties,

(3) determining the range of factors,

(4) determining the benchmark (here are the original parameters of the solenoid),

(5) calculating the sensitivity coefficient, and

(6) selecting the key factors.

Assuming that the framework of the proportional solenoid does not change, the structural size of the coil, shell cover and fixed iron core is unchanged. The parameters in Table III are selected as the analysis objects. The parameters are defined in Fig. 8.

Table III: Range of the parameters.

\begin{tabular}{|c|c|c|c|}
\hline \multicolumn{2}{|c|}{ Parameters } & Benchmark & Range \\
\hline \multirow{4}{*}{ Armature } & $L(\mathrm{~mm})$ & 27 & $25-30$ \\
\cline { 2 - 4 } & $R(\mathrm{~mm})$ & 9.35 & $9-9.4$ \\
\cline { 2 - 4 } & $S(\mathrm{~mm})$ & 1 & $0-2.4$ \\
\hline \multirow{3}{*}{$\begin{array}{c}\text { Magnetic-isolated } \\
\text { ring }\end{array}$} & $\alpha\left(^{\circ}\right)$ & 60 & $35-70$ \\
\cline { 2 - 4 } & $\beta\left(^{\circ}\right)$ & 60 & $35-70$ \\
\cline { 2 - 4 } & $I M(\mathrm{~mm})$ & 5.2 & $4-6$ \\
\hline Sleeve & $F M(\mathrm{~mm})$ & 7.3 & $6-9$ \\
\hline
\end{tabular}

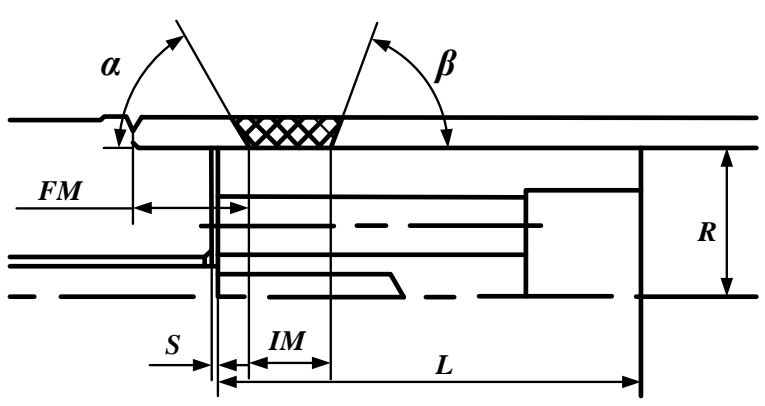

Figure 8: Definition of the parameters.

Supposing that a system exists, the system characteristic $P$ is mainly determined by $n$ factors: $a=\left\{a_{1}, a_{2}, \ldots, a_{n}\right\}, P=f\left(a_{1}, a_{2}, \ldots, a_{n}\right\}$. For a certain benchmark $a^{*}=\left\{a^{*}{ }_{1}, a^{*}{ }_{2}, \ldots, a_{n}{ }_{n}\right\}$, the system characteristic is $P^{*}$. When each factor changes within its range, the trend and range of system characteristic $P$, which deviated from the benchmark $P^{*}$, are analysed because of the changes in its related factors.

If the system characteristic $P$ is the electromagnetic force, the original structural parameter of the proportional structure is the benchmark and the armature length, armature radius, magnetic-isolated ring length and other parameters comprise the factor $a$, then sensitivity analysis of each parameter can be conducted accordingly.

When the effect of factor $a_{k}$ on characteristic $P$ is analysed, the other parameters are set to the fixed benchmark, $a_{k}$ is changed within a given range and the performance of system characteristic $P$ is expressed as:

$$
P=f\left(a_{1}^{*}, \ldots, a_{k-1}^{*}, a_{k}^{*}, a_{k+1}^{*}, \ldots, a_{n}^{*}\right)=\emptyset_{k}\left(a_{k}\right)
$$

Numerous parameters are involved; thus, the units of the parameters vary, and the sensitivity function and sensitivity factor of the dimensionless form are defined accordingly. 
The ratio of the relative error, $\delta_{k}=\left|\Delta a_{k}\right| / a_{k}$, of the system characteristic $P$ is defined as the sensitivity function $S_{k}=\left(a_{k}\right)$ of parameter $a_{k}$ :

$$
S_{k}\left(a_{k}\right) \triangleq \frac{\left(\frac{|\Delta P|}{P}\right)}{\left(\frac{\left|\Delta a_{k}\right|}{a_{k}}\right)}=\left|\frac{\Delta P}{\Delta a_{k}}\right| \frac{a_{k}}{P}, k=1,2, \ldots, n
$$

When $\left|\Delta a_{k}\right| / a_{k}$ is small, $S_{k}=\left(a_{k}\right)$ can be approximated as:

$$
S_{k}\left(a_{k}\right)=\left|\frac{d\left(\emptyset_{k}\left(a_{k}\right)\right.}{d a_{k}}\right| \frac{a_{k}}{P}, k=1,2, \ldots, n
$$

If $a_{k}=a_{k}^{*}$, then the sensitivity factor $S_{k}^{*}$ of parameter $a_{k}$ can be obtained as:

$$
S_{k}^{*}=S_{k}^{*}\left(a_{k}\right)=\left|\left(\frac{d \emptyset_{k}\left(a_{k}\right)}{d a_{k}}\right)_{a_{k}=a_{k}^{*}}\right| \frac{a_{k}^{*}}{P^{*}}, k=1,2, \ldots, n
$$

A high $S_{k}^{*}$ indicates that $P$ is more sensitive to $a_{k}$ in this benchmark. A comparative analysis of the sensitivity of the factors of the system characteristics can be conducted by comparing their sensitivity factors $S_{k}^{*}$. The orthogonal method was used for sensitivity analysis, and the values of the parameters in a given range were adjusted at evenly spaced intervals. The calculation can be completed on the Workbench platform.

\section{RESULTS AND DISCUSSION}

\subsection{Sensitivity analysis results}

The parameter sensitivity analysis results are shown in Fig. 9. The parameters are arranged according to their sensitivity.

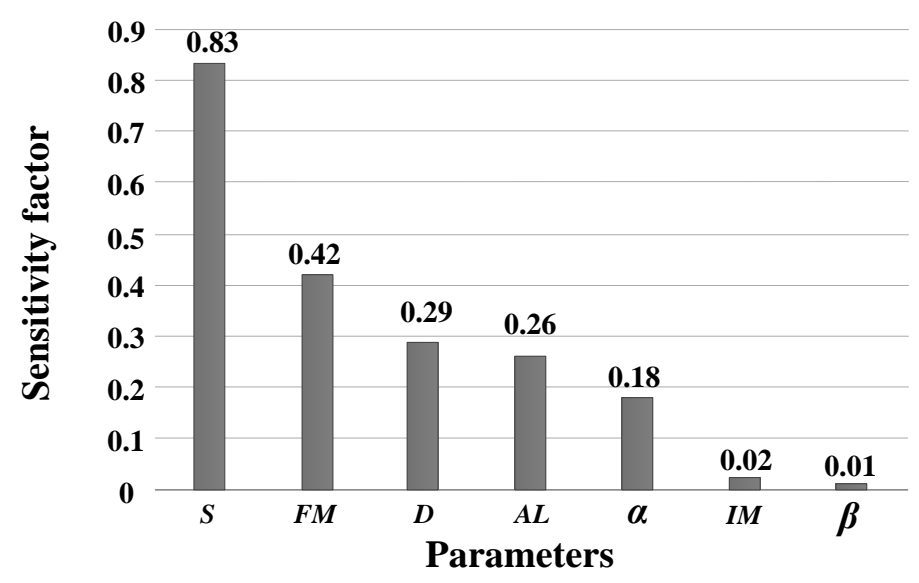

Figure 9: Parameter sensitivity analysis results.

Firstly, the armature displacement $(S)$ has the strongest effect on the electromagnetic force. This result indicates that the force-displacement characteristic of the proportional solenoid is unsatisfactory when the benchmark is used. In an ideal case, the force-displacement curve of the proportional solenoid should be a straight line, and $S$ has a minimal effect on the electromagnetic force. The sensitivity of $S$ to the electromagnetic force reflects the forcedisplacement characteristic of the proportional solenoid and shows the poor performance of the proportional solenoid, which should be improved.

Secondly, the first half sleeve width $(F M)$, armature radius $(R)$, armature length $(L)$ and magnetic-isolated ring angle $(\alpha)$ also exhibit sensitivity. By contrast, the magnetic-isolated ring width $(I M)$ and magnetic-isolated ring angle $(\beta)$ are less sensitive to the electromagnetic force; thus, both parameters are disregarded temporarily. 
In summary, $F M, R, L$ and $\alpha$ have significant effects on the electromagnetic force; thus, they can be further analysed and optimized as the key parameters.

\subsection{Analysis of the key parameters}

The static performance of the proportional solenoid is mainly reflected by the forcedisplacement curve; thus, the force-displacement curve was considered the analysis object in the subsequent analyses.

\section{First half sleeve width (FM)}

$F M$ changes from $6 \mathrm{~mm}$ to $9 \mathrm{~mm}$, and seven force-displacement curves are drawn according to the different $F M$ s, as shown in Fig. 10. When the $F M$ deviation from the benchmark is gradually reduced, the shape of the force-displacement curve becomes closer to the switching solenoid, that is, when the armature is far from the fixed iron core, the electromagnetic force decreases significantly. However, when the armature is close to the zero position, the electromagnetic force increases remarkably. When the $F M$ deviation from the benchmark increases gradually, the constant force-displacement characteristic improves. With the continuous increase in $F M$, the force-displacement curve will show an 'upturned' phenomenon, that is, the average value of the second half of the curve is greater than the average value of the first half of the curve. From the structural point of view, the change in $F M$ directly influences the location of the magnetic-isolated ring. When $F M$ is reduced, the relative distance between magnetic-isolated ring and fixed iron core and the effective displacement of the armature are reduced. Therefore, the appropriate increase in $F M$ can improve the constant forcedisplacement characteristic, increasing the effective displacement distance of the armature, whereas not significantly reducing the electromagnetic force.

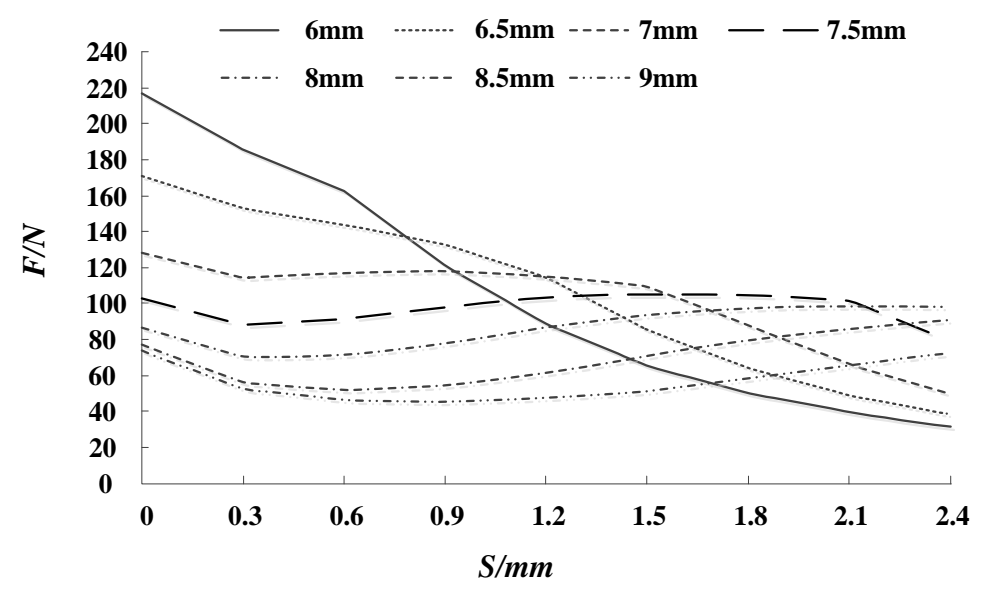

Figure 10: Influence of the first half sleeve width on the electromagnetic force.

\section{Armature radius $(\boldsymbol{R})$}

$R$ directly affects the size of the radial air gap. When the sleeve diameter is fixed, the increase in $R$ reduces the radial air gap. As shown in Fig. 11, when $R$ gradually increases from $9 \mathrm{~mm}$ to $9.4 \mathrm{~mm}$, the thickness of the radial air gap decreases from $0.5 \mathrm{~mm}$ to $0.1 \mathrm{~mm}$ and the average electromagnetic force increases continuously. With the continuous increase in $R$, the force-displacement curve upturns slowly. Therefore, increasing $R$ and reducing the thickness of the radial air gap is an effective approach to increasing the average value of the electromagnetic force. From the magnetic point of view, the reduction in the thickness of the radial air gap results in the reduction in the loss of the magnetic circuit in the air. Given cost of the actual products, $R$ should not be infinitely close to the internal radius of the sleeve; thus, comprehensive consideration is required in setting the value. 


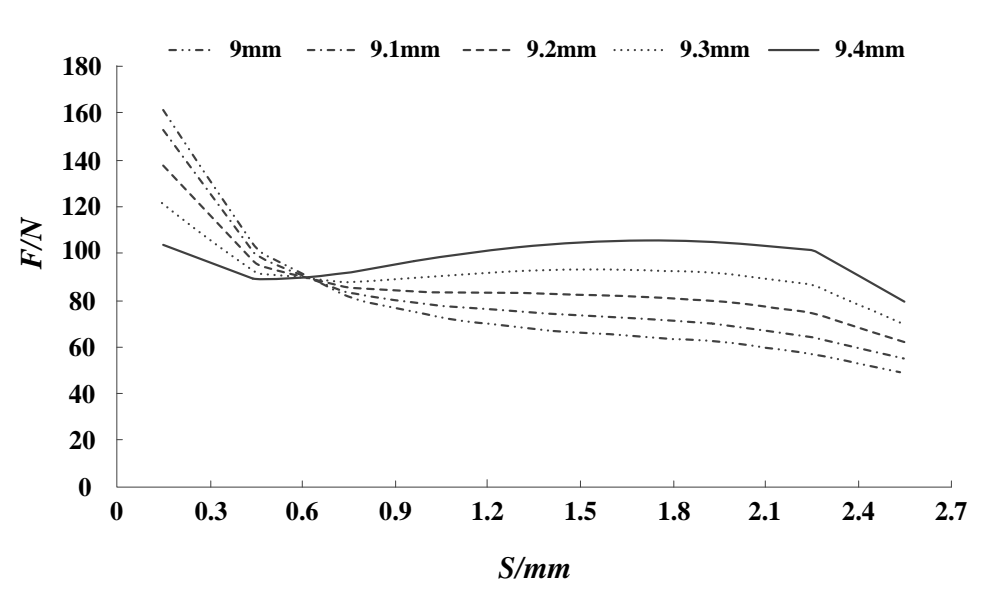

Figure 11: Influence of the armature radius on the electromagnetic force.

\section{Armature length $(L)$}

$L$ gradually increases from $25 \mathrm{~mm}$ to $30 \mathrm{~mm}$, as shown in Fig. 12. The electromagnetic force increases with the increase in $L$, but the increase value become very small after $L$ greater than $28 \mathrm{~mm}$. In summary, an increase in $L$ to a certain extent can increase the electromagnetic force; however, as length becomes longer, the increase in the electromagnetic force becomes less. Compared with the benchmark, $L$ can be increased appropriately within a limited range, because of the following reasons: (1) the increase in $L$ does not result in a significant increase in the electromagnetic force and (2) a considerable increase in the armature mass may slow down the dynamic response speed.

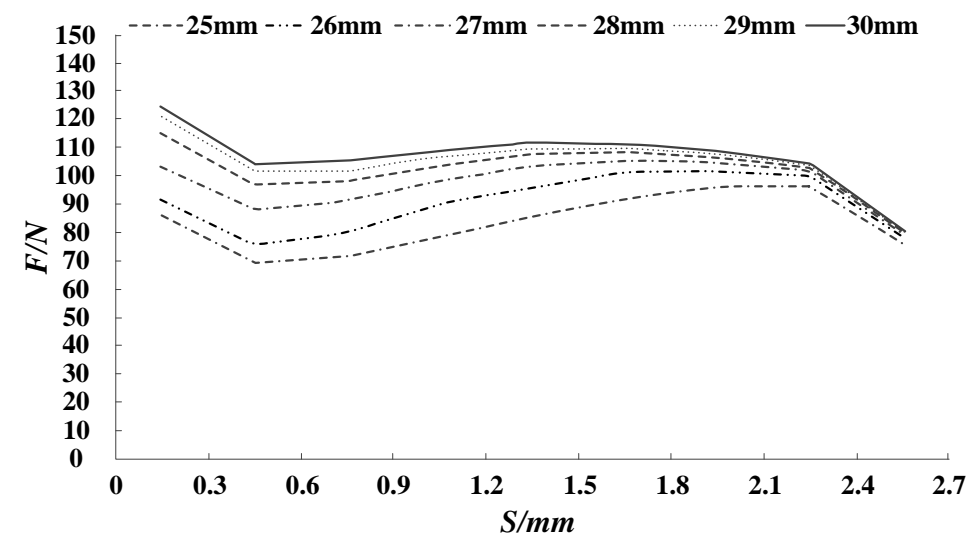

Figure 12: Influence of armature length on electromagnetic force.

\section{Magnetic-isolated ring angle ( $\alpha$ )}

The curves shown in Fig. 13 correspond to the variation in the force-displacement characteristic of $\alpha$, which increases from $35^{\circ}$ to $70^{\circ}$. The curves have a junction at $1.2 \mathrm{~mm}$ displacement and are symmetrical through this centre point. An adjustment in $\alpha$ can adjust the shape of the force-displacement curve. An increase in $\alpha$ can improve the constant forcedisplacement characteristic to some extent, but has minimal effect on the average value of the electromagnetic force within the entire stroke.

\section{Magnetic-isolated ring width (IM)}

As shown in Fig. 14, IM increased from $4 \mathrm{~mm}$ to $6 \mathrm{~mm}$, and the force-displacement curves overlapped one another. Thus, the change in the electromagnetic force is insignificant when $I M$ is increased or decreased within the vicinity of the benchmark. The simulation results are consistent with the sensitivity analysis results, verifying that $I M$ has a weak sensitivity to the electromagnetic force. 


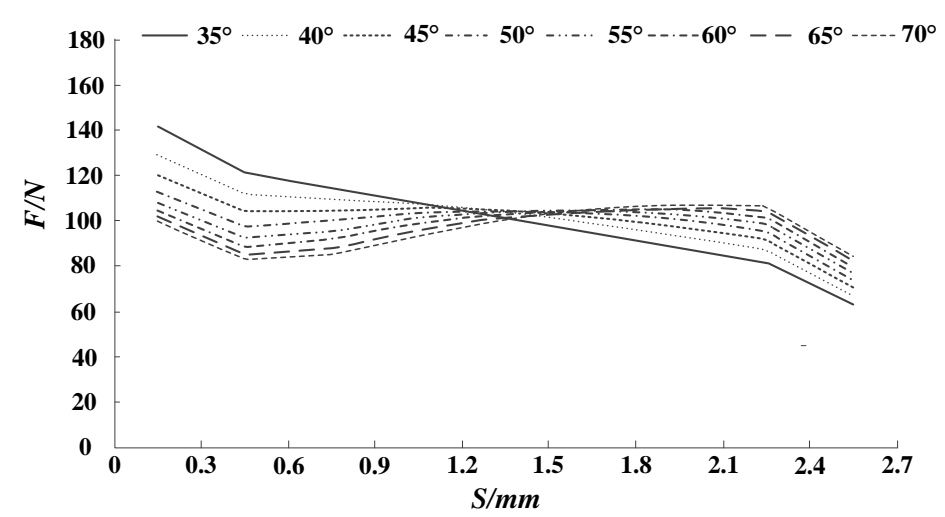

Figure 13: Influence of the magnetic-isolated ring angle $\alpha$ on electromagnetic force.

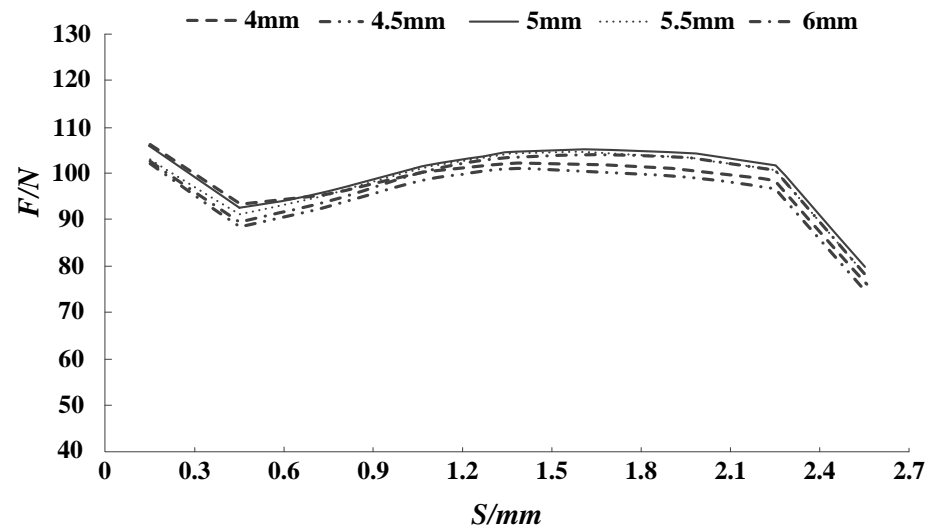

Figure 14. Influence of magnetic-isolated ring width on electromagnetic force

\subsection{Parameters optimization and experimental verification}

According to the simulation results from Figs. 10 to 14, FM should be increased to enhance the force of the second half curve, $R$ should be expanded to the maximum value within the possible scope of processing to improve the overall electromagnetic force, $L$ and $\alpha$ should be adjusted to maintain the constant force-displacement characteristic. The parameters with lower sensitivity can be remained unchanged temporarily. After debugging, a set of parameters was obtained and they are shown in Table IV. As shown in Fig. 15, when the current is $2.5 \mathrm{~A}$, the optimized parameters generally enhanced the electromagnetic force at every point. According to the simulation results, the electromagnetic force is enhanced and the effective stroke is extended to $2.1 \mathrm{~mm}$.

A new solenoid with optimized parameters was built and tested in the experimental platform shown in section 2.2. The experimental results before and after optimization is shown in Fig. 16. After optimization, the electromagnetic force is increased by an average of $20.1 \%$ within the working stroke, and the effective stroke is extended from $1.8 \mathrm{~mm}$ to $2.1 \mathrm{~mm}$ at the rated current $2.5 \mathrm{~A}$.

Table IV: Structure parameter optimization.

\begin{tabular}{|c|c|c|c|}
\hline \multirow{2}{*}{ Parameter } & \multicolumn{3}{|c|}{ Data } \\
\cline { 2 - 4 } & Original & Optimized & Change \\
\hline$F M(\mathrm{~mm})$ & 7.3 & 7.6 & +0.3 \\
\hline$D(\mathrm{~mm})$ & 9.35 & 9.45 & +0.1 \\
\hline$L(\mathrm{~mm})$ & 27 & 29 & +2 \\
\hline$\alpha\left(^{\circ}\right)$ & 60 & 55 & -5 \\
\hline$\beta\left(^{\circ}\right)$ & 70 & 70 & 0 \\
\hline$I M(\mathrm{~mm})$ & 5.2 & 5.2 & 0 \\
\hline
\end{tabular}




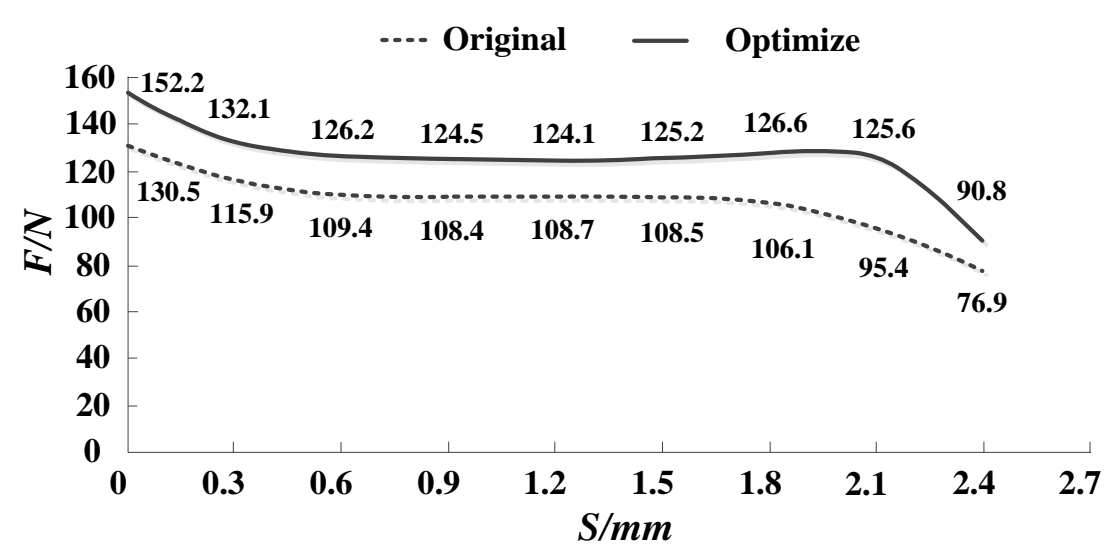

Figure 15: Simulation results with optimized and original parameters.

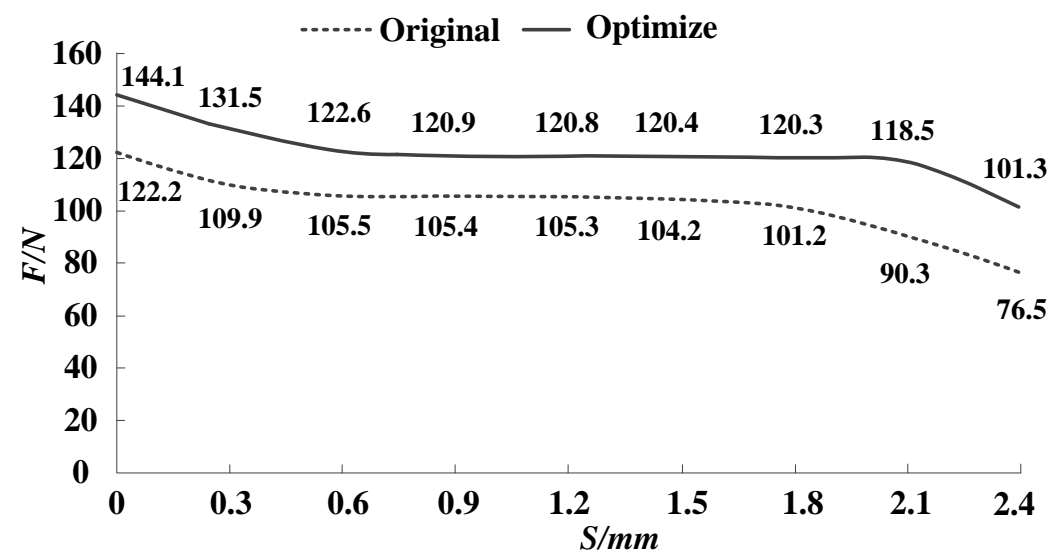

Figure 16: Experimental results with optimized and original parameters.

\section{CONCLUSIONS}

Optimization of the structural parameters by using the proposed optimization method combining 3D finite element simulation and parameter sensitivity analysis, the forcedisplacement characteristics of the proportional solenoid can be significantly improved. The main conclusions are listed in the following:

Parameter sensitivity analysis can reduce the number of parameters significantly. The result showed that the key parameters are first half sleeve width, armature radius, armature length and magnetic-isolated ring angle $\alpha$. By contrast, magnetic-isolated ring width and magnetic-isolated ring angle $\beta$ are less sensitive to the electromagnetic force.

The electromagnetic force of the proportional solenoid and the effective stroke can be increased effectively by adjusting the key parameters. After optimization, the electromagnetic force increased by an average of $20.1 \%$ and the effective stroke extended by $16.7 \%$. In addition, the constant force-displacement characteristic has also been slightly improved.

\section{ACKNOWLEDGEMENT}

Supported by National Key Research and Development Program of China (Project No. 2018YFD0700703) and Natural Science Foundation of China (Grant No. 51975538).

\section{REFERENCES}

[1] Ding, C.; Ding, F.; Zhou, X.; Liu, S.; Yang, C. Y. (2013). Novel pressure-resistant oil-immersed proportional actuator for electrohydraulic proportional control valve, Journal of Mechanical Design, Vol. 135, No. 12, Paper 125001, 5 pages, doi:10.1115/1.4025194 
[2] Zhu, Y.; Gao, X.-R. (2013). Relationship between the forces on the spool and anti-cavitation performance and throttling effect after the notch of the valve, Journal of Applied Sciences, Vol. 13, No. 10, 1677-1683, doi:10.3923/jas.2013.1677.1683

[3] Hu, S.-Y.; E, J.-Q.; Zuo, Q.-S.; Gong, J.-K. (2012). Characteristic simulation of high speed solenoid valve in diesel engine based on adaptive mutative scale chaos optimization algorithm, Journal of Central South University (Science and Technology), Vol. 43, No. 4, 1325-1330

[4] Li, Q. P. (2005). Research on the Key Technologies of Direct Drive Servo Valve, PhD Thesis, Zhejiang University, Hangzhou

[5] Zhang, L. W.; Xu, Y. M. (2013). FEM analysis for magnetic force of proportion electro-magnet, Machine Tool \& Hydraulics, Vol. 41, No. 17, 169-170, doi:10.3969/j.issn.1001-3881.2013.17.049

[6] Angadi, S. V.; Jackson, R. L.; Choe, S.-Y.; Flowers, G. T.; Suhling, J. C.; Chang, Y.-K.; Ham, J.K. (2009). Reliability and life study of hydraulic solenoid valve - Part 1: a multi-physics finite element model, Engineering Failure Analysis, Vol. 16, No. 3, 874-887, doi:10.1016/ j.engfailanal.2008.08.011

[7] Tao, G.; Chen, H. Y.; J, Y. Y.; He, Z. B. (2002). Optimal design of the magnetic field of a highspeed response solenoid valve, Journal of Materials Processing Technology, Vol. 129, No. 1-3, 555-558, doi:10.1016/S0924-0136(02)00633-7

[8] Zhou, X. T.; Liu, H. Z.; Qiu, R.; Liu, L. L.; Yuan, D. N. (2013). Non-contact loading of high speed motorized spindle, Journal of Central South University (Science and Technology), Vol. 44, No. 7, 2756-2763

[9] Sun, Z.-Y.; Li, G.-X.; Wang, L.; Wang, W.-H.; Gao, Q.-X.; Wang, J. (2016). Effects of structure parameters on the static electromagnetic characteristics of solenoid valve for an electronic unit pump, Energy Conversion and Management, Vol. 113, 119-130, doi:10.1016/ j.enconman.2016.01.031

[10] Liu, P.; Fan, L. Y.; Qaisar, H.; Xu, D.; Ma, X. Z.; Song, E. Z. (2014). Research on key factors and their interaction effects of electromagnetic force of high-speed solenoid valve, The Scientific World Journal, Vol. 2014, Paper 567242, 13 pages, doi:10.1155/2014/567242

[11] Man, J.; Ding, F.; Li, Q. P.; Da, J. (2010). Novel high-speed electromagnetic actuator with permanent-magnet shielding for high-pressure applications, IEEE Transactions on Magnetics, Vol. 46, No. 12, 4030-4033, doi:10.1109/TMAG.2010.2078826

[12] Zhang, Q. (2009). Finite element analysis of electromagnetic force of proportional electromagnet based on Ansoft, Journal of Shenyang Normal University (Natural Science Edition), Vol. 27, No. 3, 306-309

[13] Li, W.; Hu, X.-D.; Li, Q.-P. (2013). Simulation analysis of high-speed on-off solenoid returned by permanent magnetic force, Journal of Mechanical \&Electrical Engineering, Vol. 30, No. 4, 444446

[14] Billenstein, D.; Dinkel, C.; Reig, F. (2018). Automated topological clustering of design proposals in structural optimisation, International Journal of Simulation Modelling, Vol. 17, No. 4, 657-666, doi:10.2507/IJSIMM17(4)454

[15] Ningbo Hoyea Machinery Manufacture Co., Ltd. HOYEA, from http://www.hoyea.com, accessed on 15-06-2020

[16] Cao, J. N.; Liu, J.; Yang, Y. (2012). Impact characteristics study on electromagnet magnetic isolation ring to electromagnetic force, Applied Mechanics and Materials, Vol. 201-202, 635-639, doi:10.4028/www.scientific.net/AMM.201-201.635

[17] Zeng, X. T.; Meng, G. Y.; Zheng, K. (2019). Force transmission analysis of sliding block-type hydraulic support under impact loads, International Journal of Simulation Modelling, Vol. 18, No. 1, 100-111, doi:10.2507/IJSIMM18(1)466

[18] Encica, L.; Paulides, J. J. H.; Lomonova, E. A. (2008). Passive and active constant forcedisplacement characteristics and optimization of a long-stroke linear actuator, Proceedings of the $11^{\text {th }}$ International Conference on Optimization of Electrical and Electronic Equipment, 117-124, doi:10.1109/OPTIM.2008.4602397 